\title{
Role Of Relationship Style In Delaying Help Seeking From An Oncologist In Women With Ovarian Cancer
}

$\underline{\text { S. Datta }}^{1}$, L. Fraser ${ }^{2}$, M. Burnell ${ }^{3}$, S. Nasreen ${ }^{1}$, M. Ghosh ${ }^{1}$, A. Ojha ${ }^{1}$, T. Saha ${ }^{1}$, A. Mukhopadhaya ${ }^{4}$, A. Lanceley ${ }^{2}$, U. Menon'

${ }^{1}$ Tata Medical Center, Palliative Care and Psycho-oncology, Kolkata, India.

${ }^{2}$ University College London, Institute for Women's Health, London, United Kingdom.

${ }^{3}$ University College London, MRC Clinical Trials Unit at UCL, London, United Kingdom.

${ }^{4}$ Tata Medical Center, Department of Gynaecological Oncology, Kolkata, India.

Objectives: The present study explores that role of relationship styles to the time to present to an oncologist for women with ovarian cancer.

Background and Aims: Health related help seeking behaviour may be affected by adult attachment and relationship style. Majority of women with ovarian cancer in India present in advanced stages of the disease. The present study aimed at exploring the relationship of attachment style on the time to help seeking from an oncologist in adult women with ovarian cancer.

Materials and Methods: One hundred thirty-two consecutive patients with ovarian cancer presenting to a tertiary cancer centre were recruited for the study and were interviewed using pre-designed socio-demographic data, disease related questionnaire, Hospital Anxiety Depression Scale (HADS), Rosenburg Self-esteem Scale (RSES) and Experiences of Close Relationships - Relationship Style questionnaire (ECR-RS). ECR-RS quantifies the relationship style in adults.

Results: The median time from the onset of symptoms to being seen by a cancer specialist was 3 (IQR1.25 - 5) months. On Hospital Anxiety Depression Scale (HADS), the median anxiety score of our study sample was 8 (IQR $4.25-12.75)$ and median depression score was 7 (IQR 5-12). In multivariate

analysis, belonging to a joint family [Mean ratio $1.5,95 \% \mathrm{Cl}$ 1.01-2.33, $p=0.04]$ and having increased ECR Anxiety subscale score [Mean ratio 1.16, 95\% Cl 1.02-1.3, $p=0.02$ ] were both independently associated with delay in presentation to oncologist.

Conclusion: Increased attachment related anxiety and belonging to a joint family is associated with delayed help seeking from an oncologist in women with ovarian cancer.
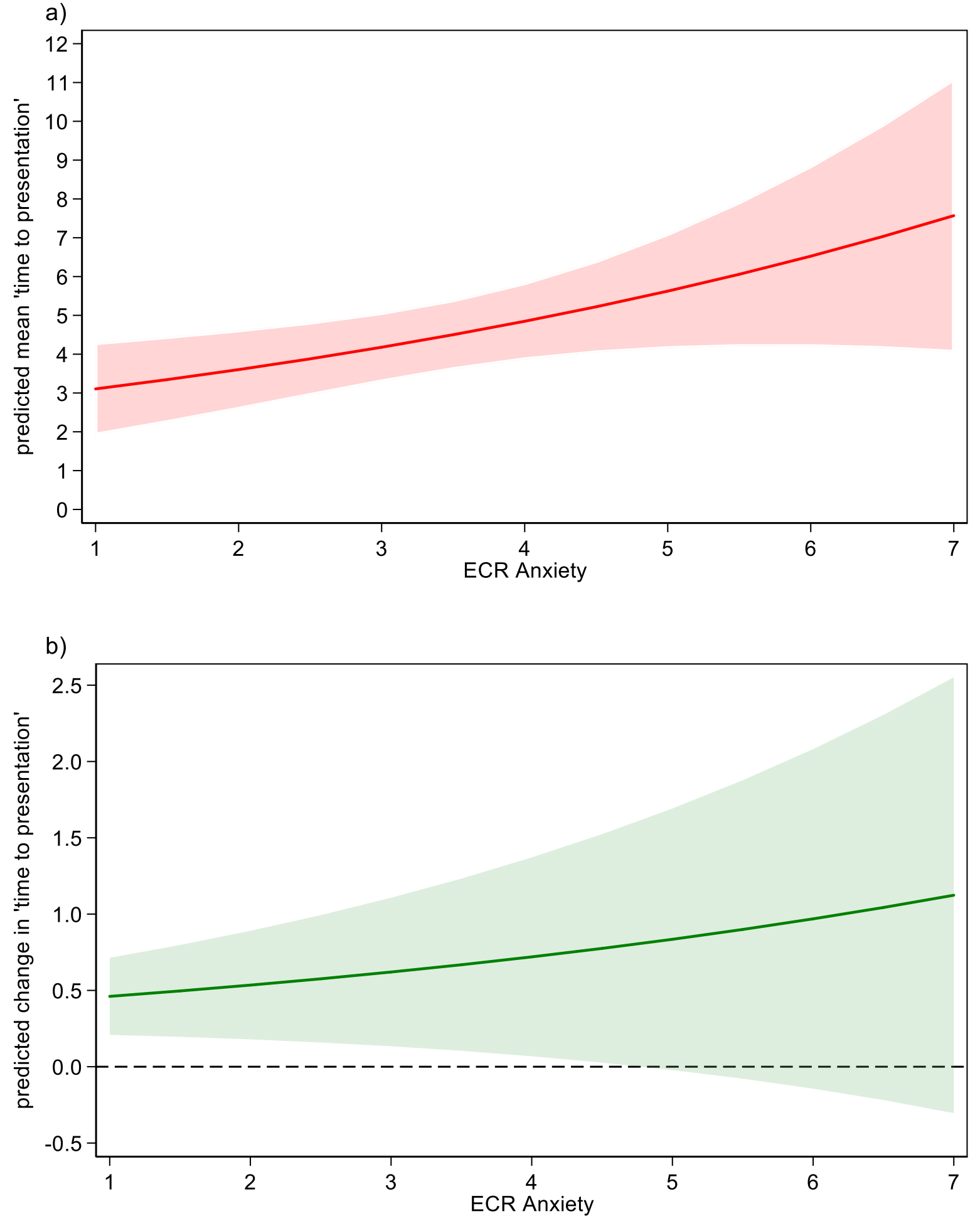

Fig 1. Attachment pattern and time to presentation in women with ovarian cancer

Keywords: attachment style, ovarian cancer, depression, delay, family 